\title{
Inactivation de gènes et fonction
}

\author{
Jean-Pierre Zanetta
}

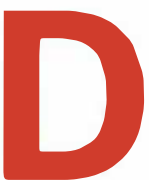

ans un numéro récent de médecine/sciences $\left(n^{\circ} 5\right.$ vol. 10, p. 565), Matthieu Lévi-Strauss s'est interrogé d'une façon extrêmement pertinente sur la signification de résultats inattendus (souvent négatifs), obtenus lors d'expériences d'inactivation de gènes de certaines molécules considérées comme fondamentales dans le fonctionnement ou la mise en place correcte d'un organisme. Il est évident que, face à de tels résultats négatifs, on s'interroge sur les possibilités de compensation du mécanisme supprimé. C'est l'argument le plus souvent avancé, bien que, malgré de très nombreuses études, rien de semblable n'ait été démontré dans les cas discutés. Est-ce que la technique, discutable en elle-même, n'induit pas le système de compensation? Mais avant de s'interroger sur l'existence de mécanismes non encore imaginables, il conviendrait de se poser la question de savoir si les fonctions biologiques envisagées pour ces molécules considérées comme essentielles ont réellement été démontrées. Les exemples choisis dans l'article de médecine/sciences sont particulièrement intéressants à cet égard.

L'auteur de cet article, citant l'article de Rutishauser [1], considère que, pour la N-CAM, "de très classiques expériences ont pourtant montré le rôle crucial dans l'adhérence entre neurones embryonnaires, ou entre neurones et cellules musculaires". Si quelques dizaines de milliers de chercheurs s'escri- tiquer le rôle de cette molécule, n'en déplaise à G. Edelman, il n'existe aucun rôle véritablement démontré de cette molécule dans l'adhérence cellulaire. En 1988, Rutishauser [2] a lui-même proposé un autre rôle (anti-adhésif) pour la $\mathrm{N}$-CAM, réfutant ses premiers arguments.

Les arguments en faveur de l'implication de cette molécule dans l'adhérence cellulaire proviennent essentiellement des effets inhibiteurs sur l'adhérence cellulaire des anticorps dirigés contre la N-CAM. La validité de ces travaux reste toutefois douteuse car les quantités d'anticorps utilisées sont bien trop grandes pour assurer une quelconque spécificité. En fait, et cela n'a pas été analysé sérieusement par les auteurs, des quantités similaires d'anticorps (ou de fragments Fab des anticorps) dirigés contre des molécules sans rapport avec le phénomène analysé ont, comme effet primaire, d'être cytotoxiques. Il n'est donc pas étonnant qu'un effet soit observé. Mais est-il véritablement spécifique et, en conséquence, peuton déduire réellement de ces expériences les conclusions généralement tirées ?

Avant d'invoquer des mécanismes inconnus pour justifier ces échecs retentissants, il serait sans doute plus logique de s'interroger d'abord sur le bien-fondé des protocoles expérimentaux qui ont conduit à proposer de tels rôles pour ces molécules. Si la plupart des scientifiques du domaine de l'adhérence cellulaire acceptent ce rôle de la N-CAM et la notion d'interaction homophile pro- posée il y a vingt-deux ans, l'hypothèse des rôles de la N-CAM et des molécules supposées agir dans l'adhérence cellulaire par interactions homophiles a été critiquée [3, $4]$. La lecture attentive des protocoles expérimentaux et des résultats publiés dans la littérature indique plutôt que la N-CAM n'est pas une molécule essentielle dans les contacts entre cellules nerveuses. Des mécanismes alternatifs ont été proposés, selon lesquels ce sont certains variants de glycosylation très mineurs de la N-CAM et des molécules de la même famille qui participent à l'adhérence, au même titre que d'autres glycoprotéines plus abondantes, qui pourraient jouer un rôle essentiel. L'hypothèse alternative (glycobiologique) est que des glycannes de ces glycoprotéines sont reconnus par des lectines endogènes. En pontant ces glycannes de surface (lectines polyvalentes solubles et extracellulaires) ou en reconnaissant un glycanne à la surface d'une cellule en vis-à-vis (lectines membranaires), les lectines expliquent comment les glycannes de ces glycoprotéines peuvent intervenir dans l'adhérence et la reconnaissance [5]. Plusieurs données de la littérature, provenant de groupes travaillant sur les CAM, indiquent que certains glycannes jouent, en effet, un rôle majeur dans les phénomènes adhésifs dans le système nerveux [6]. Inactiver le gène de la $\mathrm{N}$ CAM revient à éliminer peut-être une partie pour dix mille des capacités adhésives des neurones immatures, et une partie pour cent des activités d'ATPase dépendant du 
$\mathrm{Ca}^{2+}$ et $\mathrm{du} \mathrm{Mg}^{2+}$ (puisque la N-CAM possède cette activité [7]). Dans l'optique glycobiologique des interactions cellulaires, il était donc prévisible que l'inactivation du gène de la N-CAM ne pût significativement perturber le développement d'un animal. Les résultats de cette expérience étaient donc attendus avec impatience par les partisans de l'hypothèse glycobiologique.

$\mathrm{Au}$ moment où cet échec sur la $\mathrm{N}$ CAM était relaté, étaient publiés les résultats de l'inactivation du gène de la $\mathrm{N}$-acétyl-glucosaminyltransférase I, une enzyme importante dans la biosynthèse des $\mathrm{N}$-glycannes des glycoprotéines. L'inactivation de ce gène nécessaire à la production de glycannes de type complexe et hybride produit des glycannes oligomannosidiques et provoque une accumulation de N-glycannes de type Man4GlcNAc2 (qui ne sont pas reconnus par des lectines endogènes). Toutes les souris meurent à mi-gestation, avec une absence de fermeture du tube neural [8]. Ce résultat était attendu puisque, in vitro, les cellules mutantes $\mathrm{CHO}$ déficientes en cette enzyme ne sont plus adhérentes, qu'elles soient ou non transfectées avec les gènes des CAM [9]. Certes, s'il est difficile, à l'heure actuelle, d'aller très loin dans l'interprétation des résultats de cette expérience d'invalidation génique, on peut néanmoins en conclure que les N-glycannes complexes et hybrides ne sont pas nécessaires aux premiers stades de développement de l'embryon, mais le deviennent lors de la fermeture du tube neural ; au cours de cette période, certains auteurs ont suggéré que les séquences polysialosyl $\alpha 2-8$ et HNK-1*, que l'on trouve sur des glycoprotéines impliquées dans l'adhérence cellulaire, étaient importantes. Cela plaide en faveur d'un rôle fondamental des N-glycannes portés par certaines glycoprotéines, dont la NCAM, mais suggère, encore, que la $\mathrm{N}$-CAM reste un élément extrêmement mineur et peu important dans l'adhérence cellulaire.

Les hypothèses glycobiologiques ont elles-mêmes connu des échecs. C'est le cas de l'absence d'effets [10] de

\footnotetext{
* HNK-1 : groupement acide glucuronique 3sulfate.
}

l'inactivation du gène de la lectine spécifique des lactosides L-14, bien que le rôle de cette lectine dans la fusion des myoblastes (rôle antiadhésif) ait été élégamment montré in vitro [11]. Mais dans ce cas, la compensation paraît possible puisqu'il existe une famille de lectines spécifiques des lactosides ayant des gènes différents [12] et que l'une des propriétés caractéristiques de ces lectines est d'être extrêmement inductible.

Il existe donc, sans doute, des cas où l'inactivation de gènes va induire des systèmes de compensation qui vont rendre la mutation inefficace. Mais l'absence d'effets d'une expérience de ce type présente souvent un intérêt fondamental pour le biologiste. Elle permet de s'interroger (enfin) sur la fonction biologique réelle de la molécule en question, de remettre en question des hypothèses fonctionnelles aux bases expérimentales parfois précaires et ne reposant, souvent, que sur l'accès facile de certains auteurs aux grandes revues scientifiques

\section{RÉFÉRENCES}

1. Rutishauser U, Grumet M, Edelman GM. Neural cell adhesion molecule mediates initial interactions between spinal cord neurons and muscle cells in culture. J Cell Biol 1983 ; 97 : 145-52.

2. Rutishauser U, Acheson A, Hall AK, Mann DM, Sunshine J. The neural cell adhesion molecule (N-CA ) as a regulator of cell interactions. Science $1988 ; 352$ : 53-7.

3. Zanetta JP. Is N-CAM a non-cell adhesion molecule ? Glycobiology $1991 ; 1$ : 317-20.

4. Zanetta JP. A re-evaluation of the concept of homophilic interactions? Glycobiology 1994 (sous presse).

5. Zanetta JP, Kuchler S, Lehmann S, Badache A, Maschke S, Thomas D, Dufourcq P, Vincendon G. Glycoproteins and lectins in cell adhesion processes. Histochem J 1992 ; 24 : 791-804.

6. Fahrig T, Schmitz B, Weber D, KuchererEhret A, Faissner A, Schachner M. 2 monoclonal antibodies recognizing carbohydrate epitopes on neural adhesion molecules interfere with cell interactions. EurJ Neurosci $1990 ; 2$ : 153-61.
7. Dzhandzhugazyan K, Bock E. Demonstration of $\left(\mathrm{Ca}^{2+}-\mathrm{Mg}^{2+}\right)$-ATPase activity of the neural cell adhesion molecule. FEBS Lett 1993 ; 336 : 279-83.

8. Ioffe E, Stanley P. Mice lacking Nacetylglucosaminyltransferase I activity die at mid-gestation, revealing an essential role for complex or hybrid N-linked carbohydrates. Proc Natl Acad Sci USA 1994 ; $91: 728-32$

9. Filbin MT, Tennekoon GI. The role of complex carbohydrates in adhesion of the myelin protein PO. Neuron 1991 ; 7 : 84555.

10. Poirier F, Robertson EJ. Normal development of mice carrying a null mutation in the gene encoding the L14 Stype lectin. Development $1993 ; 119$ : 1229-36.

11. Cooper DNW, Massa SM, Barondes SH. Endogenous muscle lectin inhibits myoblast adhesion to laminin. J Cell Biol $1991: 115: 1437-48$.

12. Mehrabian M, Gitt MA, Sparkes RS, Leffler H, Barondes $\mathrm{SH}$, Lusis AJ. 2 members of the S-Lac lectin gene family, LGALS1 and LGALS2, reside in close proximity on human chromosome-22q12q13. Genomics $1993: 15: 418-20$.

\section{Jean-Pierre Zanetta}

Directeur de recherche au CNRS, laboratoire de biologie moléculaire des interactions cellulaires, CNRS UPR A0416, 5 , rue Blaise-Pascal, 67000 Strasbourg, France.

\section{TIRÉS A PART}

J.P. Zanetta. 